\title{
On the critical pair theory in $\mathbb{Z} / p \mathbb{Z}$
}

\author{
by \\ Yahya Ould Hamidoune (Paris), Oriol Serra (Barcelona) \\ and Gilles ZÉmor (Paris)
}

\section{Introduction}

1.1. Context and main result. The Cauchy-Davenport theorem $[2,3]$ states that if $A$ and $B$ are subsets of $\mathbb{Z} / p \mathbb{Z}$ then

$$
|A+B| \geq \min (p,|A|+|B|-1) .
$$

Vosper's theorem [19] solves the related critical pair problem and states that, if $|A|,|B| \geq 2$, then

$$
|A+B| \geq \min (p-1,|A|+|B|)
$$

unless both $A$ and $B$ are arithmetic progressions with a common difference. The Cauchy-Davenport theorem was generalized to general abelian groups by several authors including Mann [15, 16] and Kneser [13]. Kemperman proposed in [12] a recursive procedure which generalizes Vosper's theorem to all abelian groups.

In the case when the group is $\mathbb{Z}$, the analogue of Vosper's theorem was considerably strengthened. A theorem of Freiman [5] states that if a subset $A$ of integers is such that $|A+A|$ is sufficiently small then it must be contained in a short arithmetic progression: specifically, if $|A+A| \leq 2|A|+m$ with $-1 \leq m \leq|A|-4$, then $A$ must be contained in an arithmetic progression of length $|A|+m+1$.

In the case of the sum of two sets, Frelman's theorem was generalized by Lev and Smeliansky; a version of it states (see [14, Lemma 1] or [17]):

Theorem 1. Let $A, B \subset[0, b]$, where $b \in \mathbb{N}$ with $0 \in A \cap B$ and $b \in B$. Assume moreover $\operatorname{gcd}(B)=1$. Then

$$
|A+B| \geq \min (b+|A|,|A \cup(A+b)|+|B|-2) .
$$

Going back to the case of $\mathbb{Z} / p \mathbb{Z}$, Freimman proved [4], using trigonometric sums, that for a subset $A \subset \mathbb{Z} / p \mathbb{Z},|A+A| \leq 2.4|A|$ and $|A| \leq p / 35$ hold only

2000 Mathematics Subject Classification: Primary 11P70. 
if $A$ is contained in a short arithmetic progression. Bilu, Lev and Ruzsa [1] show that, for $|A|$ small enough, $|A+A| \leq 3|A|-4$ holds only if $A$ is a subset of a short arithmetic progression. It is also conjectured that the result should hold without the restriction on the size of $|A|$. We will refer to this as the $(3 k-4)$-conjecture in $\mathbb{Z} / p \mathbb{Z}$.

Going beyond Vosper's theorem without any undue restriction on the size of $A$ and $B$ has proved challenging. A recent result of Rødseth and one of the present authors [11] is a step in that direction.

For a subset $X$ of an abelian group, let $\ell_{r}(X)$ denote, if it exists, the cardinality of the smallest arithmetic progression with difference $r$ containing $X$. We have [11]:

Theorem 2. Let $A, B$ be subsets of $\mathbb{Z} / p \mathbb{Z}$ with $|A| \geq 3$ and $|B| \geq 4$. If

$$
|A+B| \leq|A|+|B| \leq p-4
$$

then $\ell_{r}(A) \leq|A|+1$ and $\ell_{r}(B) \leq|B|+1$ for some $r \in \mathbb{Z} / p \mathbb{Z}$.

The above results suggest the following conjecture.

ConjeCture 1 . Let $m$ be a non-negative integer and let $A, B$ be subsets of $\mathbb{Z} / p \mathbb{Z}$ such that

$$
|A+B| \leq|A|+|B|+m \leq p-(m+4) .
$$

If $|A| \geq m+3$ and $|B| \geq m+4$ then there is $r \in \mathbb{Z} / p \mathbb{Z}$ such that

$$
\ell_{r}(A) \leq|A|+m+1, \quad \ell_{r}(B) \leq|B|+m+1 .
$$

The conditions of Conjecture 1 cannot be weakened. For example, take $A=\{0,1, \ldots, m+1\} \cup\{2 m+4+j\}$ for any positive integer $j$; we have $|2 A|=2|A|+m$ for large enough $p$ and $A$ is not contained in an arithmetic progression of the stated length. Now let $B=\mathbb{Z} / p \mathbb{Z} \backslash(-2 A)$; then it can be easily checked that $|A+B|=p-|-A|=|B|+|A|+m$, which shows that the condition $|A+B| \leq p-(m+4)$ cannot be removed.

Note that the choice $m=|A|-4$ in Conjecture 1 gives the $(3 k-4)$ conjecture in $\mathbb{Z} / p \mathbb{Z}$ for sets $A$ with $|A| \leq(p-1) / 4$. The best known result, due to Green and Ruzsa [6], only implies the validity of this $(3 k-4)$ conjecture when $|A|<10^{-180} p$.

The case $m=0$ of Conjecture 1 is Theorem 2. In the present paper we extend Theorem 2 to the case $m=1$. More precisely, we prove:

Theorem 3. Let $A, B$ be subsets of $\mathbb{Z} / p \mathbb{Z}$ with $|A| \geq 4$ and $|B| \geq 5$. If $p \geq 53$ and

$$
|A+B| \leq|A|+|B|+1 \leq p-5,
$$

then there is $r \in \mathbb{Z} / p \mathbb{Z}$ such that

$$
\ell_{r}(A) \leq|A|+2, \quad \ell_{r}(B) \leq|B|+2 .
$$


1.2. Methodology: isoperimetric tools and outline of the paper. Let $G$ be an abelian group and let $B \subset G$ be a generating subset of $G$ such that $0 \in B$. Let $k$ be a positive integer; the $k$ th isoperimetric number of $B$ is

$$
\kappa_{k}(B)=\min \{|X+B|-|X|:|X| \geq k \text { and }|X+B| \leq|G|-k\},
$$

where $\min \emptyset=|G|$, by convention. A subset $X$ achieving the above minimum is called a $k$-fragment of $B$. A $k$-fragment with minimal cardinality is called a $k$-atom.

In the context of additive problems, atoms were first introduced in [7] and have since proved useful tools in critical pair theory (see e.g. $[8,9,10]$ ).

It is proved in [7] that for any abelian group $G$ and any subset $B \subset G$, a 1-atom of $B$ containing 0 is a subgroup. This result easily implies Mann's generalization of the Cauchy-Davenport theorem. The structure of 2-atoms is more difficult to describe. For $\kappa_{2}(B) \leq|B|$, it is proved in [10, Theorem 6.2] that a 2 -atom of $B$ containing 0 with size $\geq 3$ is a subgroup. A critical pair theory is deduced from this description in [10]. 2-atoms were again used in [11] as an essential tool in the proof of Theorem 2. The structure of 2 -atoms in $\mathbb{Z} / p \mathbb{Z}$ was further studied by the authors of [18]. As a consequence it is shown that every set $B \subset \mathbb{Z} / p \mathbb{Z}$ is the union of $h=\kappa_{2}(B)-|B|+2$ arithmetic progressions with the same difference provided that $|B| \leq p-(h+2)^{2} / 2$. This could be seen as a partial result on the way to Conjecture 1: to cover the remaining ground one would need in particular to bound the gaps between consecutive arithmetic progressions.

In the present paper we depart from previous work by making use for the first time of $k$-atoms for $k>2$. We envisage the following isoperimetric method to deal with Conjecture 1: it comes in three steps.

- Step 1: Prove a special case of Conjecture 1 assuming that one of the two sets $A$ and $B$ is already known to be contained in a short arithmetic progression.

- Step 2: Replace the hypotheses of Conjecture 1 by the weaker conditions $\kappa_{m+4}(A) \leq|A|+m$ and $\kappa_{m+3}(B) \leq|B|+m$.

- Step 3: Study an $(m+3)$-atom $K$ of $A$ and an $(m+4)$-atom $L$ of $K$. It is enough to prove the result for $L$, since a repeated application of Step 1 allows one to recover the structure of $A$ and $B$.

Before trying this approach for large values of $m$, we think that better understanding of the structure of the $k$-atoms is required. Indeed, since Conjecture 1 contains the long-standing $(3 k-4)$-conjecture for $\mathbb{Z} / p \mathbb{Z}$, one may suspect that the above program will not be without technical difficulties. However, to check the soundness of the proposed method we shall try it out in this paper by considering the first open case $m=1$ of Conjecture 1 . 
The paper is organised as follows. In Section 2 we give some preliminary results which allow us to give a first bound on the size of $k$-atoms of a set $B$ for $k \leq|B|$. In Section 3 we show that if a pair of sets satisfy the conditions of Theorem 3 and one of the two sets is contained in a short arithmetic progression then so is the second one. We next show in Section 4 that 4 atoms of a set $B$ with $\kappa_{5}(B) \leq|B|+1$ have cardinality 4 and that when $|B|=4$ then the 5 -atoms of $B$ have cardinality 5 . Section 5 presents the remaining ingredient of the proof which consists of saying that, when the two sets in Theorem 3 are small, they are indeed contained in a short arithmetic progression. Finally, the proof of Theorem 3 is completed in Section 6 .

2. A first bound on the size of atoms. In this section we introduce notation and preliminary results which will be used throughout the paper. We also derive a preliminary bound on the size of $k$-atoms that we will need.

Two subsets $X$ and $Y$ of $\mathbb{Z} / p \mathbb{Z}$ will be said to be equivalent if there is $u \neq 0$ and $v$ such that $Y=u \cdot X+v$, where $u \cdot X=\{u x: x \in X\}$. Note that the $k$-isoperimetric numbers of a set and the fact of being a $k$-fragment or a $k$-atom are invariant properties under translations and automorphisms of the additive group $\mathbb{Z} / p \mathbb{Z}$. Therefore there is no loss of generality in considering equivalent sets.

We recall known results that we shall use.

Lemma 4 ([9, Lemma 2.4]). Let $B$ be a subset of a finite abelian group $G$. Let $F$ be a k-fragment of $B$ and $a \in G$. Then $a-F$ and $G \backslash(F+B)$ are $k$-fragments of $-B$. Moreover $\kappa_{k}(-B)=\kappa_{k}(B)$.

The following is a particularly useful property of $k$-atoms.

Theorem 5 ([9, Proposition 2.5]). Let $B$ be a subset of a finite abelian group $G$. Let $M$ be a $k$-atom of $B$. Let $F$ be a $k$-fragment of $B$ such that $M \not \subset F$. Then $|M \cap F| \leq k-1$.

Throughout the paper we use the following notation. Let $X$ and $Y$ be subsets of $\mathbb{Z} / p \mathbb{Z}$. For each integer $i \geq 0$ we introduce the set $N_{i}(X, Y)$ defined by

$$
\begin{aligned}
& N_{0}(X, Y)=X, \\
& N_{1}(X, Y)=(X+Y) \backslash X, \\
& N_{i}(X, Y)=(X+i Y) \backslash(X+(i-1) Y), \quad i \geq 2,
\end{aligned}
$$

where $i Y=Y+\cdots+Y$ (i times). We simply write $N_{i}$ when the reference to sets $X$ and $Y$ is clear from the context.

For a subset $U$ of $Y$ and $i \geq 1$ such that $N_{i} \neq \emptyset$, we denote by $N_{i}^{U}$ the set of elements $z \in N_{i}$ such that $z-U \subset N_{i-1}$ and $(z-(Y \backslash U)) \cap N_{i-1}=\emptyset$. 
We also write

$$
N_{i}^{\subseteq U}=\bigcup_{V \subseteq U} N_{i}^{V} .
$$

Lemma 6. Let $X, Y \subset \mathbb{Z} / p \mathbb{Z}, U \subset Y$ and $i \geq 1$. With the notation just introduced, if $N_{i+1}^{U} \neq \emptyset$ then

$$
N_{i+1}^{U}-U \subset N_{i}^{\subseteq U}
$$

In particular,

$$
\left|N_{i+1}^{U}\right| \leq\left|N_{i}^{\subseteq U}\right|-|U|+1
$$

Proof. Let $z \in N_{i+1}^{U}, u \in U$ and $z^{\prime}=z-u \in N_{i}$. Then $z^{\prime} \in N_{i}^{V}$ for some subset $V$ of $Y$. But, for any $v \in V$, we have $z-v=z^{\prime}-v+u \in N_{j}$ for some $j<i+1$. Since $z \in N_{i+1}$ we must have $j=i$; this implies $V \subset U$. In particular, if $N_{i+1}^{U} \neq \emptyset$, then

$$
N_{i+1}^{U}-U \subseteq \bigcup_{V \subset U} N_{i}^{V}=N_{i}^{\subseteq U} .
$$

By the Cauchy-Davenport theorem, $\left|N_{i+1}^{U}-U\right| \geq\left|N_{i+1}^{U}\right|+|U|-1$.

We will use the following result originally obtained in [18]. We provide here a shortened proof. We use the notation $\bar{X}$ as shorthand for $\mathbb{Z} / p \mathbb{Z} \backslash X$.

TheOREM 7 ([18]). Let $B$ be a subset of $\mathbb{Z} / p \mathbb{Z}$ containing 0 and let $A$ be a 2-atom of $B$ containing 0 . Set $m=\kappa_{2}(B)-|B|=|B+A|-|B|-|A|$. Assume that $|B|<p-(m+4)(m+3) / 2$. Then $|A|=2$. In particular $B$ is a union of at most $m+2$ arithmetic progressions with the same difference.

Proof. Suppose that $|A|>2$. Set $A^{*}=A \backslash\{0\}$. Let us first show that

$$
N_{i+1}-A^{*} \subset N_{i}, \quad i \geq 1
$$

where $N_{i}=N_{i}(B, A)$. Take $x \in N_{2}$. Since $x-A$ meets $N_{1}$, we see that $x-A$ is not a subset of $\overline{B+A}$. By Lemma $4, x-A$ and $\overline{B+A}$ are respectively a 2-atom and a 2-fragment of $-B$. Theorem 5 implies $(x-A) \cap \overline{B+A}=\{x\}$ or, equivalently, $x-A^{*} \subset N_{1}$. We have just proved (1) for $i=1$. The assertion follows for $i>1$ by Lemma 6 since $N_{2}^{U}=\emptyset$ for each proper subset $U \subset A^{*}$.

Let $t$ be the largest integer such that $N_{t} \neq \emptyset$. Since $A$ generates $\mathbb{Z} / p \mathbb{Z}$, we have

$$
\mathbb{Z} / p \mathbb{Z}=B \cup N_{1} \cup N_{2} \cup \cdots \cup N_{t}, \quad t \geq 2 .
$$

By (1) and Lemma 6 we have

$$
\left|N_{i+1}\right| \leq\left|N_{i}\right|-(|A|-2), \quad 1 \leq i<t,
$$

and $\left|N_{1}\right|=|B+A|-|B|=|A|+m$. 
Suppose first that $t \geq 3$. Using (2) and $|A| \geq 3$, we get

$$
\begin{aligned}
p & \leq|B|+(m+|A|)+(m+2)+(m+4-|A|)+\sum_{i=0}^{m-1}(m-i) \\
& \leq|B|+\sum_{i=1}^{m+3} i=|B|+(m+4)(m+3) / 2,
\end{aligned}
$$

contradicting the assumption on $|B|$.

Suppose now that $t=2$. In this case $\overline{B+A}=N_{2}$. Since $\overline{B+A}$ is a 2-fragment of $-B$, we have $|A| \leq|-(\overline{B+A})|=\left|N_{2}\right|$; furthermore, $\left|N_{2}\right| \leq m+2$ by (2). We therefore obtain $p \leq|B|+(m+|A|)+(m+2)<$ $|B|+(m+4)(m+3) / 2$, again a contradiction.

Theorem 7 provides us with a useful bound of the size of $k$-atoms.

Proposition 8. Let $B$ be a subset of $\mathbb{Z} / p \mathbb{Z}$ containing 0 and let $A$ be a $k$-atom of $B$ with $2 \leq k \leq|B|$. Put $m=\kappa_{k}(B)-|B|$. Assume moreover $p+k>m^{2}+6 m+12$. Then $|A| \leq m+k+1$.

Proof. We may assume $0 \in A$. By definition of $m$ we have $|B+A|=$ $|B|+|A|+m$. Let $C=\overline{B+A}$ so that $p=|A|+|B|+|C|+m$. By definition of $k$-atoms we have $|C| \geq k$ and $|A| \geq k$. Observe that $(C-B) \cap A=\emptyset$ so that $|B-C|=|C-B| \leq|B|+|C|+m$ and therefore $|A| \leq|C|$ by the minimality condition in the definition of $k$-atoms. Hence, $2|A| \leq|A|+|C|=p-|B|-m$. The condition $k \leq|B|$ now implies

$$
\begin{aligned}
|A| & \leq(p-k-m) / 2 \\
& <\left(2 p-m^{2}-6 m-12-m\right) / 2=p-(m+4)(m+3) / 2 .
\end{aligned}
$$

Now $|B+A|=|B|+|A|+m$ implies that $\kappa_{k}(A)-|A| \leq m$ and hence that $\kappa_{2}(A)-|A| \leq m$. Therefore Theorem 7 implies that $A$ is a union of not more than $m+2$ arithmetic progressions with the same difference $u$. This gives

$$
|A|-m-2 \leq|A \cap(A+u)| \text {. }
$$

Furthermore, since $A+u$ is also a $k$-atom of $B$, Theorem 5 implies

$$
|A \cap(A+u)| \leq k-1 \text {, }
$$

hence the result.

3. Compression transfer. Let us start with a lemma which is the $\mathbb{Z}$-counterpart of our main result.

Lemma 9. Let $A$ and $B$ be subsets of $\mathbb{Z}$ such that $0 \in A \cap B$ and

$$
|A+B| \leq|A|+|B|+1, \quad|B| \geq|A| \geq 4,|B| \geq 5 .
$$

Then $A$ and $B$ have the same greatest common divisor $r=\operatorname{gcd}(A)=\operatorname{gcd}(B)$ and $\ell_{r}(A) \leq|A|+2$ and $\ell_{r}(B) \leq|B|+2$. 
Proof. Let $r=\operatorname{gcd}(A)$. Put $B_{1}=\{x \in B: x=0 \bmod r\}$ and $B_{2}=$ $B \backslash B_{1}$. We have $B_{1} \neq \emptyset$ since $0 \in B_{1}$ and if $\operatorname{gcd}(B)<r$ then $B_{2} \neq \emptyset$ also. We then must have $|A+B| \geq|A|+\left|B_{1}\right|-1+|A|+\left|B_{2}\right|-1 \geq|A|+|B|+2$, a contradiction. This proves $\operatorname{gcd}(B) \geq \operatorname{gcd}(A)$. Proceed likewise to obtain $\operatorname{gcd}(A) \geq \operatorname{gcd}(B)$.

We may assume $A, B \subset \mathbb{N}$ and $\operatorname{gcd}(A)=\operatorname{gcd}(B)=1$ without loss of generality. Put $a=\max (A)$ and $b=\max (B)$.

Assume first $a \geq b$. Apply Theorem 1 with $A$ and $B$ interchanged to get

$$
|A+B| \geq \min (a+|B|, 2|B|-1+|A|-2) .
$$

Hence $|A|+|B|+1 \geq \min (a+|B|,|A|+|B|+(|B|-3))$, and since $|B| \geq 5$ we must have $|A|+|B|+1 \geq a+|B|$, which means $\ell_{1}(A) \leq|A|+2$. Since $|A| \leq|B|$ and $\max (B) \leq \max (A)$ we clearly must also have $\ell_{1}(B) \leq|B|+2$.

Suppose now $a<b$. Apply Theorem 1 to get

$$
|A+B| \geq \min (b+|A|, 2|A|+|B|-2) .
$$

Since $|A| \geq 4$ we get $|A|+|B|+1 \geq b+|A|$, which means $\ell_{1}(B) \leq|B|+2$. Notice furthermore that if $|B| \leq|A|+1$ then $a<b$ implies $\ell_{1}(A) \leq|A|+2$.

Summarizing, we have proved that under the hypothesis of the lemma, $\ell_{1}(B) \leq|B|+2$ always holds and $\ell_{1}(A) \leq|A|+2$ holds under the additional condition

$$
|B| \leq|A|+1 .
$$

We proceed to prove by induction on $|B|$ that under the hypothesis of the lemma $\ell_{1}(A) \leq|A|+2$ always holds. If $|B|=5$ then (3) must hold and we are done. Now suppose by induction that the result holds for $|B|=\beta \geq 5$, and consider the case $|B|=\beta+1$. Let $B^{\prime}=B \backslash\{b\}$. Since the result holds with (3) assume $|A| \leq\left|B^{\prime}\right|$. Since $|B|=\beta+1 \geq 6$ we have $\left|B^{\prime}\right| \geq 5$. Since $a+b \notin A+B^{\prime}$ we also have $\left|A+B^{\prime}\right| \leq|A|+\left|B^{\prime}\right|+1$. The result follows from the induction hypothesis applied to $A$ and $B^{\prime}$.

The aim of this section is to show that, under the hypothesis of Theorem 3 , if one of the two sets is in a short arithmetic progression with difference $r$, then so is the other one.

TheORem 10. Let $X, Y$ be subsets of $\mathbb{Z} / p \mathbb{Z}$ such that

$$
|X+Y|=|X|+|Y|+1 \leq p-5
$$

and with $|Y| \geq 4,|X| \geq 5$. If $p>32$ then $\ell_{1}(Y) \leq|Y|+2$ implies $\ell_{1}(X) \leq$ $|X|+2$.

The proof of Theorem 10 will be broken down into several lemmas.

First, we need some notation. By a connected component of a set $Z \subset$ $\mathbb{Z} / p \mathbb{Z}$ we mean a maximal arithmetic progression of difference 1 contained 
in $Z$. Let $C_{1}, \ldots, C_{j}$ be the connected components of the complement $\bar{X}$ of $X$ with $\left|C_{j}\right|=\max _{1 \leq i \leq j}\left|C_{i}\right|$. Thus, $\ell_{1}(X)=p-\left|C_{j}\right|$. We have

$$
|X|+|Y|+1=|X+Y|=|X|+\sum_{i=1}^{j}\left|(X+Y) \cap C_{i}\right| .
$$

For $i=1, \ldots, j$ we shall use the notation $C_{i}=\left\{c_{i}, \ldots, c_{i}+\left|C_{i}\right|-1\right\}$, and $X_{i}$ denotes the connected component of $X$ containing $c_{i}-1$. We assume that $j>1$ since otherwise there is nothing to prove.

Lemma 11. Theorem 10 holds if $\left|C_{j}\right| \geq \ell_{1}(Y)-1$.

Proof. $\left|C_{j}\right| \geq \ell_{1}(Y)-1$ means that by translation we may choose $Y \subset$ $\left[0, \ell_{1}(Y)-1\right]$ and $X \subset\left[0, \ell_{1}(X)-1\right]$ with $\ell_{1}(X)+\ell_{1}(Y)-1 \leq p$. In other words $X+Y$ can in fact be considered as a sum in $\mathbb{Z}$ and the result follows from Lemma 9.

Lemma 12. Theorem 10 holds if $\left|C_{j}\right|<\ell_{1}(Y)-1$ and $\ell_{1}(Y) \leq|Y|+1$.

Proof. Suppose that $\ell_{1}(Y)=|Y|$. Then $\left|(X+Y) \cap C_{i}\right|=\left|C_{i}\right|$, which implies $X+Y=\mathbb{Z} / p \mathbb{Z}$ against the assumptions.

Suppose now that $\ell_{1}(Y)=|Y|+1$. Let $Y_{1}, Y_{2}$ be the connected components of $Y$ with $y_{i}=\left|Y_{i}\right|$. By using multiplication by -1 and translating if necessary, we may assume that $Y_{1}=\left\{0, \ldots, y_{1}-1\right\}, Y_{2}=\left\{y_{1}+1, \ldots, y_{1}+y_{2}\right\}$ and $y_{1} \geq y_{2}$.

We have

$$
\left|(X+Y) \cap C_{i}\right|=\left\{\begin{array}{l}
\left|C_{i}\right|, \text { or } \\
\left|C_{i}\right|-1 \geq\left|Y_{1}\right|-1 \text { and } c_{i}-2 \in \bar{X},
\end{array}\right.
$$

which implies $j \geq|\overline{X+Y}|$ and, by using (4),

$$
\begin{aligned}
|Y|+1 & =\sum_{i=1}^{j}\left|(X+Y) \cap C_{i}\right| \geq|\overline{X+Y}|\left(\left|Y_{1}\right|-1\right) \geq 5\left(\left|Y_{1}\right|-1\right) \\
& \geq|Y|+3\left|Y_{1}\right|-5 .
\end{aligned}
$$

Hence $\left|Y_{1}\right|=2$ and all equalities hold. In particular, $X$ consists of $|\overline{X+Y}|$ $=5$ connected components each of cardinality one and $\left|C_{i}\right|=2$ for each $i$, which implies that $p$ is divisible by 3 , a contradiction.

In the remaining part of this section we assume that $\ell_{1}(Y)=|Y|+2$. Let $Y=Y_{1} \cup Y_{2} \cup Y_{3}$ be the decomposition of $Y$ into connected components, with $Y_{2}$ possibly empty, $y_{i}=\left|Y_{i}\right|$ and $y_{1}=\max _{i} y_{i}$. By using multiplication by -1 and/or translating $Y$ if necessary, we shall assume that $Y_{1} \cup Y_{2} \subset$ $\left\{0,1, \ldots, y_{1}+y_{2}\right\}$.

LEMma 13. Theorem 10 holds if $\left|C_{j}\right|<\ell_{1}(Y)-1$ and either $|Y|>9$ or $|\overline{X+Y}| \geq 12$. 
Proof. Since $\left|C_{i}\right|<\ell_{1}(Y)-1$ we have $\left|\left(X_{i}+Y\right) \cap C_{i}\right| \geq\left|C_{i}\right|-2$ and, if equality holds, then $\left|C_{i}\right| \geq\left|\left(\left(c_{i}-1\right)+Y\right) \cap C_{i}\right| \geq\left|Y_{1}\right|+\left|Y_{2}\right|-1$. Moreover, $c_{i}-2 \in \bar{X}$. By using similar remarks when $\left|\left(X_{i}+Y\right) \cap C_{i}\right|=\left|C_{i}\right|-1$ we have

$$
\left|(X+Y) \cap C_{i}\right|=\left\{\begin{array}{l}
\left|C_{i}\right|, \text { or } \\
\left|C_{i}\right|-1 \geq\lceil|Y| / 3\rceil-1 \text { and }\left|X_{i}\right| \leq 2, \text { or } \\
\left|C_{i}\right|-2 \geq\lceil|Y| / 2\rceil-1 \text { and }\left|X_{i}\right|=1 .
\end{array}\right.
$$

Suppose first that $|Y| \geq 10$. For $r=0,1,2$ let $J_{r} \subset J=\{1, \ldots, j\}$ be such that $\left|(X+Y) \cap C_{i}\right|=\left|C_{i}\right|-r$. We have $\left|J_{1}\right|+2\left|J_{2}\right|=|\overline{S+T}| \geq 5$. Therefore, using (4) and (6), we get

$$
|Y|+1=\sum_{i=1}^{j}\left|(X+Y) \cap C_{i}\right| \geq\left|J_{0}\right|+\left|J_{1}\right|(\lceil|Y| / 3\rceil-1)+\left|J_{2}\right|(\lceil|Y| / 2\rceil-1),
$$

which can hold only if $\left|J_{0}\right|=0,\left|J_{1}\right|=1$ and $\left|J_{2}\right|=2$ (and $|Y|=10$ or 12). In this case (6) implies that $X$ consists of two connected components of cardinality one and one connected component of cardinality at most two, against the assumption that $|X| \geq 5$.

Suppose now that $|Y| \leq 9$ and $|\overline{X+Y}| \geq 12$. Then (6) implies that the connected components of $\overline{X+Y}$ have cardinality 1 or 2 . If at most one has cardinality 2, then $|J|>10$. On the other hand, since $|Y| \geq 4$ we have $\left|Y_{1}\right| \geq 2$ and $\left|(X+Y) \cap C_{i}\right| \geq 1$ for each $i \in J$. Hence, $|X+Y|>|X|+10$, which contradicts $|X+Y|=|X|+|Y|+1$. If at least two connected components of $\overline{X+Y}$ have cardinality 2, then (6) implies that the corresponding two $C_{i}$ 's contain at least $|Y|-2$ elements of $(X+Y) \backslash X$. The remaining $C_{i}$ 's contribute at least one element to $(X+Y) \backslash X$ and there are at least $(12-4) / 2=4$ of them, meaning again that $|X+Y|>|X|+|Y|+1$.

Proof of Theorem 10. By Lemmas 11-13, the only case left to be examined is when $\ell_{1}(Y)=|Y|+2,|Y| \leq 9$ and $|\overline{X+Y}| \leq 11$. Set $X^{\prime}=\overline{X+Y}$. Note that $X^{\prime}-Y \subset \bar{X}$ so that $\left|X^{\prime}-Y\right| \leq\left|X^{\prime}\right|+|-Y|+1$. Now the condition $p>32$ implies $\left|\overline{X^{\prime}-Y}\right|=p-\left|X^{\prime}\right|-|Y|-1>11$. Therefore Lemma 13 applies to $X^{\prime}$ and $-Y$ and we obtain $\ell_{1}\left(X^{\prime}\right) \leq\left|X^{\prime}\right|+2$. Hence $X^{\prime}-Y=\bar{X}$ also satisfies $\ell_{1}(\bar{X}) \leq|\bar{X}|+2$ (easily checked). But this means that $X=\overline{\bar{X}}$ is the union of at most two single elements and of a progression $Z$ with $|Z| \geq 3$, since $|X| \geq 5$. By (6), $|Z| \geq 3$ implies $\left|J_{0}\right| \geq 1$ and since $|J| \leq 3$ we get $|X+Y| \leq 4$, a contradiction. This concludes the proof of Theorem 10. 
4. On the size of atoms. The bound on the size of $k$-atoms of a set $X$ given by Proposition 8 can be improved when $\kappa_{k}(X) \leq|X|+1$. We prove the following.

Theorem 14. Let $X \subset \mathbb{Z} / p \mathbb{Z}$ be such that $0 \in X,|X| \geq 4$ and $\kappa_{5}(X) \leq$ $|X|+1$. Suppose $p>|X|+42$. Then

(i) the 4-atoms of $X$ have cardinality 4 ,

(ii) if $|X|=4$ then the 5 -atoms of $X$ have cardinality 5 .

We shall break up the proof of Theorem 14 into several lemmas. First we introduce some convenient notation and terminology.

For $z \in N_{i}(X, Y), i \geq 1$, define its outdegree $d_{+}(z)=\left|(z+Y) \cap N_{i+1}\right|$ and its indegree $d_{-}(z)=\left|(z-Y) \cap N_{i-1}\right|$. Note that by counting in two ways the number of couples $\left(z, z^{\prime}\right)$ such that $z \in N_{i}, z^{\prime} \in N_{i+1}$ and $z^{\prime}-z \in Y$, we have

$$
\sum_{z \in N_{i}} d_{+}(z)=\sum_{z \in N_{i+1}} d_{-}(z)
$$

We shall call the quantity in (7) indifferently the total outdegree of $N_{i}$ or the total indegree of $N_{i+1}$.

Lemma 15. Suppose $A$ is a $k$-atom of some set $X \subset \mathbb{Z} / p \mathbb{Z}$. If there is $z \in X+A$ uniquely expressible as $z=x+a$ with $x \in X$ and $a \in A$, then $|A|=k$.

Proof. If $|A|>k$ then $A^{\prime}=A \backslash\{a\}$ satisfies $\left|X+A^{\prime}\right|-\left|A^{\prime}\right| \leq|X+A|-|A|$, which contradicts $A$ being a $k$-atom.

Lemma 16. Theorem 14 holds if $\ell_{r}(X) \leq|X|+2$ for some $r$.

Proof. Suppose on the contrary that $A$ is a $k$-atom of $X$ of size $|A|>$ $k \geq 4$. By Theorem 10 we have $\ell_{r}(A) \leq|A|+2$ so that the sum $X+A$ can be considered as a sum in $\mathbb{Z}$. There is therefore $z \in X+A$ uniquely expressible as $z=x+a, x \in X, a \in A$, contradicting Lemma 15.

Lemma 17. Let $X$ and $p$ be as in Theorem 14. Let $A$ be a 4-atom of $X$. Then $|A| \leq 5$.

Proof. By Proposition 8 we have $|A| \leq 6$. Suppose that $|A|=6$. We may assume that $0 \in A \cap X$. Let $A^{*}=A \backslash\{0\}$. Note that $A^{*}$ is not a $d$-progression, since otherwise $|A \cap(A+d)| \geq 4$, contradicting Theorem 5 . By Lemma 16 we may assume $\ell_{r}(X) \geq|X|+3$ for each $r \in \mathbb{Z} / p \mathbb{Z}^{*}$.

- We have $\left|N_{2}^{a b}\right|=0$ for every $a, b \in A^{*}$.

Otherwise $\left|N_{1}^{a b}\right| \geq 2$ and $A^{\prime}=A \backslash\{a, b\}$ satisfies $\left|X+A^{\prime}\right|-\left|A^{\prime}\right| \leq|X+A|-|A|$, which contradicts $A$ being a 4 -atom.

- $\left|N_{2}^{a b c}\right| \leq 1$ for every $a, b, c \in A^{*}$. 
Otherwise $\left|N_{1}^{\leq a b c}\right| \geq 4$ and $A^{\prime}=A \backslash\{a, b, c\}$ satisfies $\left|\left(X+A^{\prime}\right) \backslash X\right| \leq 3$, but then Theorem 2 implies that $\ell_{r}(X) \leq|X|+1$ for some $r$.

Lemma 6 therefore implies

- $\left|N_{3}^{a b c}\right|=0$ for every $a, b, c \in A^{*}$.

- $\left|N_{2}^{a b c d}\right| \leq 2$ for every $a, b, c, d \in A^{*}$.

Otherwise $\left|N_{1}^{\leq a b c d}\right| \geq 6$ and $\{0, e\}=A \backslash\{a, b, c, d\}$ is such that $|X+\{0, e\}| \leq$ $|X|+1$, which implies that $\ell_{e}(X)=|X|$.

- $\left|N_{3}^{a b c d}\right| \leq 3$ for every $a, b, c, d \in A^{*}$.

Otherwise $\left|N_{2}^{\leq a b c d}\right| \geq 7$ by Lemma 6 , but this contradicts $\left|N_{2}^{a b c d}\right| \leq 2$ and $\left|N_{2}^{U}\right| \leq 1$ for $|U|=3$.

Hence we get

- $\left|N_{4}^{a b c d}\right|=0$ for every $a, b, c, d \in A^{*}$, i.e. $N_{i}=N_{i}^{A^{*}}$ for $i \geq 4$.

We now bound the $\left|N_{i}\right|$ from above. Since $\left|N_{1}\right| \leq 7$, the total outdegree of $N_{1}$ is at most $7 \cdot 5=35$, which implies, since the indegree of any element of $N_{2}$ is at least 3 , that

$$
\left|N_{2}\right| \leq 11 \text {. }
$$

This means that the total outdegree of $N_{2}$ is at most $11 \cdot 5=55$, which in turn implies, since the indegree of any element of $N_{3}$ is at least 4 , that

$$
\left|N_{3}\right| \leq 13 \text {. }
$$

Since $N_{i}=N_{i}^{A^{*}}$ for $i \geq 4$ and $A^{*}$ is not an arithmetic progression, we have, by Vosper's theorem, $\left|N_{i}\right|+\left|A^{*}\right| \leq\left|N_{i}-A^{*}\right| \leq\left|N_{i-1}\right|$, which entails $\left|N_{4}\right| \leq 8$ and $\left|N_{5}\right| \leq 3$. This implies $|\bar{X}| \leq 42$.

Lemma 18. Let $X$ and $p$ be as in Theorem 14. Let $A$ be a 4-atom of $X$. Then $|A|=4$.

Proof. Suppose the contrary. We may assume $0 \in A$. By Lemma 17 we may also assume $|A|=5$. First note that $A$ cannot be an arithmetic progression of some difference $d$, since $|A \cap(A+d)|=4$ would contradict Theorem 5. Note that, if $A^{*}$ is an arithmetic progression, then $A$ is equivalent to $B=\{0,1,2,3, u\}$. If $B^{*}$ were an arithmetic progression of difference $d$ we would have $\left|B^{*} \cap\left(B^{*}+d\right)\right|=3$ and $|\{1,2,3\} \cap\{1+d, 2+d, 3+d\}| \geq 1$, implying that $B^{*}$ is an arithmetic progression of difference 1 or 2 , neither of which is possible. We may therefore assume, without loss of generality, that $A$ is a 4 -atom of $X$ containing 0 such that $A^{*}$ is not an arithmetic progression.

By Lemma 16 we may also assume that $\ell_{r}(X) \geq|X|+3$ for each $r \in$ $\mathbb{Z} / p \mathbb{Z}^{*}$. We proceed very much along the same lines as in the previous lemma.

- We have $\left|N_{1}^{a b}\right| \leq 2$ for each $a, b \in A^{*}$. 
Otherwise $|X+(A \backslash\{a, b\})| \leq|X|+3$ and Theorem 2 implies that $\ell_{r}(X) \leq$ $|X|+1$ for some $r$.

By Lemma 6 we have

- $\left|N_{2}^{a b}\right| \leq 1$ and $\left|N_{i}^{a b}\right|=0, i \geq 3$, for each $a, b \in A^{*}$.

- $\left|N_{2}^{a b c}\right| \leq 2$ for each $a, b, c \in A^{*}$.

Otherwise, with $V=\{a, b, c\}$, we have $\left|N_{2}^{V}-V\right| \geq 5$ and $|X+(A \backslash V)| \leq$ $|X|+1$, implying that $X$ is an arithmetic progression.

Lemma 6 then implies $\left|N_{3}^{V}-V\right| \leq\left|N_{2}^{V}\right|+\left|N_{2}^{a b}\right|+\left|N_{2}^{a c}\right|+\left|N_{2}^{b c}\right| \leq 5$, so that we get

- $\left|N_{3}^{a b c}\right| \leq 3$ and $\left|N_{4}^{a b c}\right| \leq 1$ for each $a, b, c \in A^{*}$.

In particular,

- $N_{i}=N_{i}^{A^{*}}$ for $i \geq 5$.

We now bound from above the number of elements in $N_{i}, i \geq 2$. Since $\left|N_{1}\right| \leq 6$ and $\left|N_{2}^{a b}\right|=1$ implies $\left|N_{1}^{a b}\right|=2$, there are at most three elements of $N_{2}$ of indegree 2. Now since the total outdegree of $N_{1}$ does not exceed $\left|N_{1}\right| \cdot\left|A^{*}\right|=6 \cdot 4=24$, we see by (7) that $\left|N_{2}\right| \leq 9$. Actually we must have

$$
\left|N_{2}\right| \leq 8
$$

because $\left|N_{2}\right|=9$ can occur only if $d_{+}(z)=4$ for every $z \in N_{1}$, which implies $\left|N_{2}\right|=\left|N_{1}+A^{*}\right|=\left|N_{1}\right|+\left|A^{*}\right|-1$ and, by Vosper's theorem, $A^{*}$ is an arithmetic progression contrary to our assumption.

Now the total outdegree of $N_{2}$ is at most $8 \cdot 4=32$, and every element of $N_{3}$ has indegree at least 3 , so that (7) implies

$$
\left|N_{3}\right| \leq 10 \text {. }
$$

Finally, $\left|N_{4}^{A^{*}}\right|+\left|A^{*}\right| \leq\left|N_{4}^{A^{*}}-A^{*}\right| \leq\left|N_{3}\right|$ implies $\left|N_{4}^{A^{*}}\right| \leq 6$. Since the sets $N_{3}^{V}$ for $|V|=3$ and $N_{4}^{V} \neq \emptyset$ are disjoint and contain at least three elements, we have $\left|N_{4} \backslash N_{4}^{A^{*}}\right| \leq 3$ and

$$
\left|N_{4}\right| \leq 9
$$

For every $i \geq 5$ we have $\left|N_{i}\right|=\left|N_{i}^{A^{*}}\right| \leq\left|N_{i-1}\right|-\left|A^{*}\right|$ so that $\left|N_{5}\right| \leq 5$ and $\left|N_{6}\right| \leq 1$. Adding up the $N_{i}$ 's we get $|\bar{X}| \leq 39$.

Lemma 18 proves point (i) of Theorem 14 . To prove point (ii) we use the following lemma.

Lemma 19. Let $X, B \subset \mathbb{Z} / p \mathbb{Z}$ be such that $|X|=4,|B| \geq 4,0 \in X \cap B$, and

$$
|X+B| \leq|X|+|B|+1 \text {. }
$$

If $p>|B|+20$, then there is an element $z \in X+B$ which can be uniquely written as $z=x+b$ with $x \in X$ and $b \in B$. 
Proof. If $B$ is an arithmetic progression of difference $d$ then $|X+B| \leq$ $|X|+|B|+1$ clearly implies $\ell_{d}(X) \leq|X|+2$, so that the sum $X+B$ can be considered as a sum in $\mathbb{Z}$, in which case the conclusion of the lemma holds. Suppose therefore that $B$ is not an arithmetic progression. Let $X^{*}=X \backslash\{0\}$. We now write $N_{i}=N_{i}(B, X)$. Suppose that there does not exist $z$ uniquely expressible as $z=x+b, x \in X, b \in B$. This implies $N_{1}^{x}=\emptyset$ for every $x \in X^{*}$.

We have $\left|N_{1}^{x y}\right| \leq 3$ for each $x, y \in X^{*}$. Otherwise $|B+(X \backslash\{x, y\})| \leq$ $|B+X|-\left|N_{1}^{x y}\right| \leq|B|+1$, implying that $B$ is an arithmetic progression.

By successively applying Lemma 6 and writing $(m)^{+}=\max \{0, m\}$, we have

$$
\begin{aligned}
\left|N_{2}\right| & =\sum_{V \subset X^{*}}\left|N_{2}^{V}\right|=\sum_{V \subset X^{*},|V|=2}\left|N_{2}^{V}\right|+\left|N_{2}^{X^{*}}\right| \\
& \leq \sum_{V \subset X^{*},|V|=2}\left(\left|N_{1}^{V}\right|-1\right)^{+}+\left(\left|N_{1}\right|-2\right) \leq 6,
\end{aligned}
$$

where the last inequality uses the fact that $\left|N_{1}^{V}\right|=3$ and $|V|=2$ occur together at most once. Similarly,

$$
\begin{aligned}
& \left|N_{3}\right| \leq \sum_{V \subset X^{*},|V|=2}\left(\left|N_{2}^{V}\right|-1\right)^{+}+\left(\left|N_{2}\right|-2\right) \leq 5, \\
& \left|N_{4}\right|=\left|N_{4}^{X^{*}}\right| \leq\left|N_{3}\right|-2 \leq 3 \\
& \left|N_{5}\right| \leq\left|N_{4}\right|-2 \leq 1 .
\end{aligned}
$$

Therefore, $p \leq|B|+\sum_{i=1}^{5}\left|N_{i}\right| \leq|B|+20$.

To prove point (ii) of Theorem 14 consider a 5-atom $B$ of $X$. By Proposition 8 we have $|B| \leq 7$, and since $p \geq 29$, Lemma 19 implies that there is $z$ uniquely expressible as $z=x+b, x \in X, b \in B$. But this contradicts Lemma 15.

5. The case of small sets. We next prove Theorem 3 when the two sets attain their minimum possible values, $|A|=4$ and $|B|=5$. We first need the following two lemmas. As in Section 3 we define a $d$-component of a set $Z \subset \mathbb{Z} / p \mathbb{Z}$ to be a maximal arithmetic progression of difference $d$ contained in $Z$. We denote by $c_{d}(Z)$ the number of $d$-components of $Z$.

Lemma 20. Let $p>23$ and let $A, B \subset \mathbb{Z} / p \mathbb{Z}$ with $|A|=4,|B|=5$ and $|A+B|=10$. Then $c_{d}(A) \leq 2$ for some $d \in \mathbb{Z} / p \mathbb{Z}$.

Proof. Without loss of generality we may suppose $0 \in A \cap B$. By Lemma 19 there exists $C \subset B,|C|=4$, such that $|A+C|<|A+B|$. By 
inclusion-exclusion,

$$
9 \geq|A+C|=\left|\bigcup_{c \in C}(A+c)\right| \geq 16-\sum_{c, c^{\prime} \in C, c \neq c^{\prime}}\left|(A+c) \cap\left(A+c^{\prime}\right)\right|,
$$

which gives

$$
\sum_{c, c^{\prime} \in C, c \neq c^{\prime}}\left|(A+c) \cap\left(A+c^{\prime}\right)\right| \geq 7>\left(\begin{array}{c}
|C| \\
2
\end{array}\right) .
$$

Hence there are two distinct elements $c, c^{\prime} \in C$ such that $\left|(A+c) \cap\left(A+c^{\prime}\right)\right| \geq$ 2 or, equivalently, $\left|A+\left\{0, c-c^{\prime}\right\}\right| \leq|A|+2$. It follows that $C$ has at most two $d$-components for $d=c-c^{\prime}$.

Lemma 21. Let $p$ be any odd prime and let $Z \subset \mathbb{Z} / p \mathbb{Z}$ with $0 \in Z$ and $|Z|<(p+9) / 4$. If $c_{d}(Z) \leq 2$ for some $d \in(\mathbb{Z} / p \mathbb{Z})^{*}$ then some affine image of $Z$ is a subset of $\{0,1, \ldots,(p-1) / 2\}$.

Proof. Suppose that no affine image of $Z$ is a subset of $\{0,1, \ldots$, $(p-1) / 2\}$. Then we must have $c_{d}(Z)=2$.

Let $A$ and $B$ be the components of $Z$, where $a=|A| \geq|B|=b$. Let $V$ and $W$ be the components of $\bar{Z}$, where $v=|V| \leq|W|$. Without loss of generality we may assume that $A$ is represented by the integers $A_{0}=$ $\{0,1, \ldots, a-1\}$ and $B$ is represented by $B_{0}=\{v+a, \ldots, v+a+b-1\}$.

Since $Z \not \subset\{0,1, \ldots,(p-1) / 2\}$, we have $v+a+b-1 \geq(p+1) / 2$. Moreover $2 v+a+b \leq p$. It follows that $(p+1) / 2-a-b+1 \leq v \leq(p-a-b) / 2$. Now $B_{0} \subset$ $[(p+1) / 2-b+1,(p+a+b) / 2-1]$. Hence $2 * B_{0} \subset[p+3-2 b, p+a+b-2]$ so that $2 * B$ is represented by a subset of $\{-2 b+3,-2 b+2, \ldots, a+b-2\}$. Since $2 * A$ is represented by a subset of $\{0,2, \ldots, 2 a\}$, we see that $2 * Z$ is represented by a subset of $\{\inf \{-2 b+3,0\}, \ldots, \sup \{a+b-2,2 a-2\}\}$. Since $a \geq b$ we infer that $2 * Z$ is represented by a subset of $\{-2 b+2, \ldots, 2 a-2\}$. Now we must have $(p+1) / 2 \leq 2 a+2 b-4=2|Z|-4$, and hence $p \leq 4|Z|-9$, a contradiction.

Lemma 22. Let $p>23$ and $A, B \subset \mathbb{Z} / p \mathbb{Z}$ with $0 \in A \cap B,|A|=4$ and $|B|=5$ and $|A+B|=10$. Then there is $r \in \mathbb{Z} / p \mathbb{Z}$ such that $\ell_{r}(A) \leq|A|+2$ and $\ell_{r}(B) \leq|B|+2$.

Proof. Assume first that $c_{d}(A+B) \leq 2$ for some $d \in \mathbb{Z} / p \mathbb{Z}$. Then by Lemma $21, Z=A+B$ is such that $\ell_{a}(Z) \leq(p-1) / 2$ for some $a$. This implies that if $z_{1}, z_{2}, z_{1}^{\prime}, z_{2}^{\prime}$ are four integers representing elements of $Z$, then $z_{1}+z_{2}=z_{1}^{\prime}+z_{2}^{\prime} \bmod p$ implies $z_{1}+z_{2}=z_{1}^{\prime}+z_{2}^{\prime}$. Since $0 \in A \cap B$ we have $A \cup B \subset Z$, therefore $A+B$ can be considered as a sum in $\mathbb{Z}$ and Lemma 9 implies the result.

Assume now that $A+B$ has at least three $x$-components for every $x \in \mathbb{Z} / p \mathbb{Z}$. By Lemma 20 we have $c_{d}(A) \leq 2$ for some $d \in \mathbb{Z} / p \mathbb{Z}$. Assume 
$c_{d}(A)=2$, otherwise there is nothing to prove. Let us show that we must have both

$$
c_{d}(A)=2 \quad \text { and } \quad c_{d}(B) \leq 2 .
$$

Suppose the contrary. Set $A=A_{1} \cup A_{2}$ be the decomposition of $A$ into d-components where $\left|A_{1}\right| \geq\left|A_{2}\right|$.

Let us first show that $\left|A_{1}\right|=2$. Suppose the contrary, i.e. $\left|A_{1}\right|=3$. Since $B$ has at least three $d$-components, we have $\left|A_{1}+B\right| \geq|B|+3+t$, where $t$ is the number of $d$-components of $A_{1}+B$. Since $A+B$ also has at least three $d$-components, we have $|A+B| \geq|B|+3+t+(3-t)=11$, a contradiction.

Now we can write $A=\{0, d\} \cup\{x, x+d\}=\{0, d\}+\{0, x\}$. Since $B$ has at least three $d$-components we have $|B+\{0, d\}| \geq|B|+3$. Now observe that $B+\{0, d\}$ has at least three $x$-components since otherwise $A+B=(B+\{0, d\})+\{0, x\}$ would have less than three $x$-components. It follows that $|A+B|=|B+\{0, d\}+\{0, x\}| \geq|B|+6$, a contradiction. This proves (8).

We may now assume $c_{d}(B)>1$, i.e. $c_{d}(B)=2$, otherwise there is nothing to prove. Let $B=B_{1} \cup B_{2}$ be the $d$-components of $B$. We have $c_{d}(A+B)=3$, since otherwise $A+B$ has four components that are necessarily $A_{1}+B_{1}, A_{1}+B_{2}, A_{2}+B_{1}, A_{2}+B_{2}$. Since $\left|A_{i}+B_{j}\right|=\left|A_{i}\right|+\left|B_{j}\right|-1$ we would get $|A+B|=2|A|+2|B|-4=14$.

Observe that since $A_{1}+B_{1}$ and $A_{1}+B_{2}$ are disjoint (because $\left|A_{1}\right|=2$ ), they must belong to distinct $d$-components, otherwise all the sets $A_{i}+B_{j}$ are disjoint and we again get $|A+B|=14$. For the same reason $A_{2}+B_{1}$ and $A_{2}+B_{2}$ must belong to distinct $d$-components.

The sets $A_{1}+B_{1}$ and $A_{2}+B_{1}$ also belong to distinct $d$-components, otherwise the common component $C$ satisfies $|C| \geq|A|+1+\left|B_{1}\right|-1=$ $|A|+\left|B_{1}\right|$. Then the other components are $A_{1}+B_{2}$ and $A_{2}+B_{2}$. It follows that $|A+B| \geq 2|A|+\left|B_{1}\right|+2\left|B_{2}\right|-2>10$. For the same reason $A_{1}+B_{2}$ and $A_{2}+B_{2}$ belong to distinct $d$-components.

The only possibility left is that $A_{1}+B_{1} \cup A_{2}+B_{2}$ merge into one component $C$. Since one of the two $d$-components of $B$ has at least three elements we have $|C| \geq 4$. The remaining components of $A+B$ have $\left|A_{1}\right|+\left|B_{2}\right|-1$ and $\left|A_{2}\right|+\left|B_{1}\right|-1$ elements, which gives $|A+B| \geq 4+|A|+|B|-2=11$, again a contradiction. This completes the proof.

6. Proof of Theorem 3. Suppose that $|A|=4$. Let $U$ be a 5 -atom of $A$. By Theorem 14 we have $|U|=5$. By Lemma 22 we have $\ell_{r}(A) \leq|A|+2$ for some $r \in(\mathbb{Z} / p \mathbb{Z})^{*}$ and by Theorem 10 we then have $\ell_{r}(B) \leq|B|+2$.

Suppose now that $|A| \geq 5$. Let $U$ be a 4 -atom of $A$. By Proposition 8 we have $|U| \leq 6$. 
If $|U|=4$, then let $V$ be a 5 -atom of $U$. By Theorem 14 we have $|V|=5$, and by Lemma 22 we have $\ell_{r}(U) \leq|U|+2$ for some $r \in(\mathbb{Z} / p \mathbb{Z})^{*}$. By Theorem 10 we then have $\ell_{r}(A) \leq|A|+2$, and again by Theorem 10 we finally have $\ell_{r}(B) \leq|B|+2$.

If $5 \leq|U| \leq 6$, then let $V$ be a 4-atom of $U$. We have $p \geq 53$, which implies $p>|U|+42$ and therefore Theorem 14 implies $|V|=4$. Let $W$ be a 5-atom of $V$. Apply Theorem 14 again to obtain $|W|=5$. Then Lemma 22 implies $\ell_{r}(V) \leq|V|+2$ for some $r \in(\mathbb{Z} / p \mathbb{Z})^{*}$. Theorem 10 applied once implies that $\ell_{r}(U) \leq|U|+2$, then applied once more implies that $\ell_{r}(A) \leq$ $|A|+2$, and applied a third time implies finally that $\ell_{r}(B) \leq|B|+2$. This concludes the proof.

\section{References}

[1] Y. F. Bilu, V. F. Lev and I. Z. Ruzsa, Rectification principles in additive number theory, Discrete Comput. Geom. 19 (1998), 343-353.

[2] A. L. Cauchy, Recherches sur les nombres, J. École Polytechnique 9 (1813), 99-116.

[3] H. Davenport, On the addition of residue classes, J. London Math. Soc. 10 (1935), 30-32.

[4] G. A. Freĭman, Foundations of a Structural Theory of Set Addition, Transl. Math. Monogr. 37, Amer. Math. Soc., Providence, RI, 1973.

[5] - The addition of finite sets I, Izv. Vyssh. Uchebn. Zaved. Mat. 1959, no. 6 (13), 202-213 (in Russian).

[6] B. Green and I. Z. Ruzsa, Sets with small sumset and rectification, arXiv:math.NT/ 0403338, v2, 28 Jan. 2005.

[7] Y. O. Hamidoune, On the connectivity of Cayley digraphs, European J. Combin. 5 (1984), 309-312.

[8] —, Subsets with small sums in abelian groups I: The Vosper property, ibid. 18 (1997), $541-556$.

[9] —, An isoperimetric method in additive theory, J. Algebra 179 (1996), 622-630.

[10] -, Some results in additive number theory I: The critical pair theory, Acta Arith. 96 (2000), 97-119.

[11] Y. O. Hamidoune and Ø. J. Rødseth, An inverse theorem mod p, ibid. 92 (2000), 251-262.

[12] J. H. B. Kemperman, On small sumsets in an abelian group, Acta Math. 103 (1960), 63-88.

[13] M. Kneser, Summenmengen in lokalkompakten abelschen Gruppen, Math. Z. 66 (1956), 88-110.

[14] V. F. Lev and P. Y. Smeliansky, On addition of two distinct sets of integers, Acta Arith. 70 (1995), 85-91.

[15] H. B. Mann, An addition theorem for sets of elements of Abelian groups, Proc. Amer. Math. Soc. 4 (1953), 423.

[16] - Addition Theorems: The Addition Theorems of Group Theory and Number Theory, Interscience, New York, 1965.

[17] M. B. Nathanson, Additive Number Theory. Inverse Problems and the Geometry of Sumsets, Grad. Texts in Math. 165, Springer, New York, 1996. 
[18] O. Serra and G. Zémor, On a generalization of a theorem by Vosper, Integers 0, paper A10, 10 pp., electronic only (2000); http://www.integers-ejcnt.org/vo10.html.

[19] A. G. Vosper, The critical pairs of subsets of a group of prime order, J. London Math. Soc. 31 (1956), 200-205, 280-282.

Yahya Ould Hamidoune

Équipe Combinatoire

Université Pierre et Marie Curie

Case 189

4 place Jussieu

75252 Paris 5, France

E-mail: yha@ccr.jussieu.fr

Gilles Zémor

École Nationale Supérieure des Télécommunications

46 rue Barrault

75634 Paris 13, France

E-mail: zemor@enst.fr
Oriol Serra

Dept. Matematica Aplicada IV Universitat Politècnica de Catalunya

Jordi Girona, 1

E-08034 Barcelona, Spain

E-mail: oserra@mat.upc.es

Received on 12.9.2003

and in revised form on 5.7.2005

(4619) 